\title{
Spectrum Availability in Indoor Locations for Opportunistic Spectrum Access in Dense Urban Scenarios
}

\author{
F. Novillo, H. Galeana, R. Ferrús, R. Agustí \\ Signal Theory and Communications Department \\ Universitat Politècnica de Catalunya (UPC), Barcelona, Spain. \\ e-mail: [fnovillo, hiram.galeana, ferrus, ramon]@tsc.upc.edu
}

\begin{abstract}
This paper analyses the possibility to exploit opportunistic spectrum access (OSA) for short-range radio communication systems within indoor locations in dense urban areas. In particular, considering the service area of a primary system devoted to providing outdoor coverage in a dense urban scenario, the percentage of indoor locations where the secondary users can reuse the primary frequency band without disturbing the primary system or being disturbed is estimated. The analysis considers heterogeneous path loss models for the primary and secondary systems encompassing the characterization of outdoor, indoor and building penetration losses. Obtained results quantify how aspects like the location of the primary network elements and the considered interference margins to protect primary transmissions impact on the spatial availability of the primary band within the interior of the buildings.
\end{abstract}

Keywords-Cognitive radio; propagation model; opportunistic spectrum access; spectrum availability; co-existence.

\section{INTRODUCTION.}

Nowadays, there is a growing concern about the inefficiencies of the static frequency allocation model that is commonly used by regulatory bodies to assign frequency bands to license holders. In this context, the development of dynamic spectrum allocation (DSA) schemes is receiving a lot of attention in the research community, and specially, those schemes referred to as opportunistic spectrum access (OSA) [1, 2]. OSA is a dynamic spectrum access model aimed at improving spectrum utilization by allowing more services/users to share the same band according to a hierarchical access structure with primary and secondary users [1]. Hence, the basic idea is to open spectrum licensed to primary users to secondary users $(S U)$ while limiting the interference perceived by primary users. This approach does not necessarily impose severe restrictions on the transmission power of secondary users, but rather on when and where they are able to re-use a primary band so that services of the primary users are not disturbed. The applicability of the OSA model is being investigated in many different contexts. As an example, the IEEE 802.22 standard is targeting to wireless regional area networks opportunistically operating on TV frequency bands. As well, one important application context is related to the usage of licensed bands for short-range communications systems. In these systems, the probability of finding spectrum holes (i.e., spatial locations and time intervals where a given band is not occupied) is higher due to the limited transmission range of such potential secondary users.

In this paper we analyze the possibility to exploit OSA for secondary short-range radio communication systems within indoor locations in dense urban areas where a primary system is assumed to provide outdoor coverage. In particular, the main contribution of the paper is the quantification of the reusable area (i.e., spatial spectrum availability) inside the buildings located in the service area of a certain primary system. The spatial reusability conditions are established based on the level of interference that the primary receiver can tolerate from a secondary transmitter as well as the level of interference experienced by the secondary receiver due to primary transmissions. Existing works already addressed the computation of the spectral availability for secondary systems $[3,4]$, focusing mainly on the impact of using directional antennas in a single primary communication link. In our analysis, we incorporate a more detailed characterization of outdoor, indoor and building penetration losses and results are given for typical deployment configurations of the primary system in a dense urban scenario.

This paper is organized as follows. Section II describes main general aspects of the type of scenarios under study. In section III, the system model and assumptions considered to assess the spectrum reuse are introduced. Then, in section IV, conditions for the computation of the reusable area and considered propagation models are discussed. Results and provided in section $\mathrm{V}$ and, finally, concluding remarks are mentioned in section VI.

\section{SCENARIO DESCRIPTION.}

Fig. 1 illustrates the type of scenarios to be analyzed. This is an urban scenario with a high density of buildings with various floors and where a high utilization of the wireless spectrum may be expected because of the important concentration of multiple and different wireless communications systems. In our analysis, we rely on a Manhattan model to account for a typical building layout. Then, in this scenario, we consider the coexistence of primary and secondary wireless communications 
systems. The former are assumed to provide outdoor coverage through the use of base stations $(B S)$ installed at the roof of the buildings and customer premise equipment (CPE) with antennas placed either on flat roofs or outside walls of the buildings (e.g., Local Multipoint Distribution Systems, fixed WiMAX solutions). As to secondary systems, we consider short range wireless communications intended to be used within the building (e.g., residential or enterprise WLAN networks).

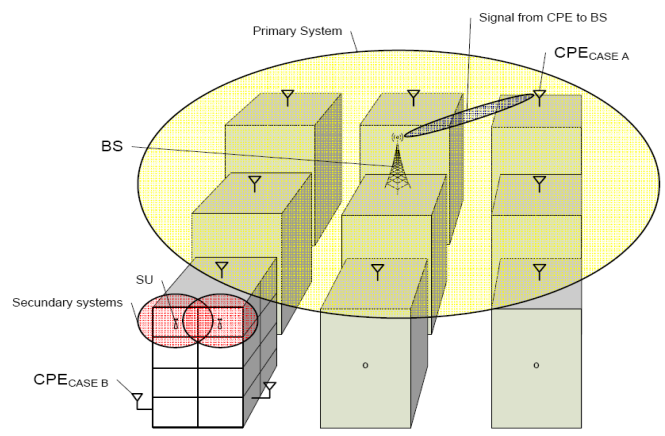

Fig. 1. Scenario under consideration.

\section{SYSTEM MODEL}

In this section we discuss the system model used to quantify spectrum reusability in aforementioned type of scenarios.

\section{A. Conditions of Spectrum Reuse.}

Conditions to determine spectrum reusability are formulated in terms of the interference levels allowed in both primary and secondary receivers. Hence, as shown in Fig.2, denoting as $I_{S P}$ the interference level from a secondary transmitter in a primary receiver, $S_{P}$ the primary receiver sensitivity and $M_{P}$ is the primary receiver protection margin, the condition that enables the usage of the primary receiver without being disturbed is given by $I_{S P} \leq S_{P}-M_{P}$ Likewise, an analogous condition follows for the successful operation of the secondary receiver: $I_{P S} \leq S_{S}-M_{S}$. In both cases, the receiver protection margins would account for the shadowing margin $\left(M_{S H}\right)$ and the interference margin $\left(M_{I}\right)$.

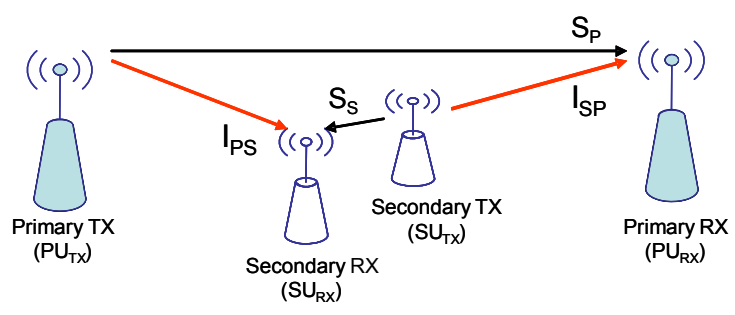

Fig. 2. Interference characterization for spectrum reuse.

\section{B. Primary System Characterization}

We consider a point-to-multipoint primary system where BSs are located at the top of buildings. Concerning the location of CPEs, two different cases are analyzed:
- Case A: CPEs are also installed at the flat roofs of the buildings and LOS (Line-of-Sight) to the BSs is required.

- Case B: CPEs are installed below building roofs in external walls or windows of the buildings. In this case Non LOS operation is considered.

Directional antennas are considered in both cases and the transmission power is dimensioned according to typical link budget parameters considered for 802.16 systems [5]. Accordingly, the BS transmission power $P_{B S}$ is calculated as follows:

$$
P_{B S}=S_{C P E}+L_{(B S-C P E)}-G_{B S}-F_{B S}(\theta)-G_{C P E}-F_{C P E}(\theta)+M_{S H}
$$

where $S_{C P E}$ is the sensibility of a CPE, $L_{(B S-C P E)}$ is the propagation loss between the BS and the considered CPE, $G_{B S}$ and $G_{C P E}$ are, respectively, the BS and CPE antenna gains, $F_{B S}(\theta)$ and $F_{C P E}(\theta)$ are, respectively the BS and CPE antenna responses with respect to the azimuth angle $\theta$ and $M_{S H}$ is the shadowing margin. Analogously, the transmitted power of a given $\mathrm{CPE}, P_{C P E}$, is given by:

$$
P_{C P E} \geq S_{B S}+L_{(C P E-B S)}-G_{C P E}-F_{C P E}(\theta)-G_{B S}-F_{B S}(\theta)+M_{S H}
$$

\section{Secondary System Characterization.}

As for secondary systems, we consider short range radio communication devices. The type of antennas used in these environments is omnidirectional and it is assumed that secondary devices always transmit at their maximum power level. Hence, no dimensioning is needed under such conditions and typical transmission parameters (transmission power, sensitivity) for 802.11 systems are used to characterize such secondary systems [5].

\section{Computation of the Reusable AREA.}

We define the reusable area as the set of locations inside the buildings where secondary devices would be able to successfully use primary spectrum. Accordingly, next we describe the necessary conditions to compute the reusable areas as well as the propagation models used in the proposed cases.

\section{A. Calculation of the Reusable Area.}

The computation of the reusable area is done by developing the two basic conditions stated in Section II.A for spectrum reuse in all indoor locations of the considered scenario. Hence, a location belongs to the reusable service area if the following four conditions are fulfilled:

$C_{l}$ : Condition intended to assert whether the interference from primary $\mathrm{BS}$ to secondary users $\left(I_{1}\right)$ is tolerable:

$$
I_{1} \triangleq P_{B S}+G_{B S}+F_{B S}(\theta)-L_{p}+G_{S U}+F_{S U}(\theta) \leq S_{S U}-M_{S U}
$$


where, $L_{P}$ is the propagation loss, $G_{S U}$ is the SU antenna gains, $F_{S U}(\theta)$ is the SU antenna responses with respect to the azimuth angle $\theta, S_{S U}$ is the sensibility of a $\mathrm{SU}, M_{S U}$ is the secondary receiver protection margin.

$C_{2}$ : Condition intended to assert whether the interference from primary CPEs to secondary users $\left(I_{2}\right)$ is tolerable:

$$
I_{2} \triangleq P_{C P E}+G_{C P E}+F_{C P E}(\theta)-L_{p}+G_{S U}+F_{S U}(\theta) \leq S_{S U}-M_{S U}
$$

$C_{3}$ : Condition intended to assert whether the interference from secondary users to primary BS $\left(I_{3}\right)$ is tolerable:

$$
I_{3} \triangleq P_{S U}+G_{S U}+F_{S U}(\theta)-L_{p}+G_{B S}+F_{B S}(\theta) \leq S_{B S}-M_{B S}
$$

\section{so, $P_{S U}$ is the SU transmission power.}

$C_{4}$ : Condition intended to assert whether the interference from secondary users to primary CPEs $\left(I_{4}\right)$.

$$
I_{4} \triangleq P_{S U}+G_{S U}+F_{S U}(\theta)-L_{p}+G_{C P E}+F_{C P E}(\theta) \leq S_{C P E}-M_{C P E}
$$

The four conditions are checked in all indoor locations of the scenario under study. As to mathematical notation, each indoor location is named as $u_{i}$ and the reusability condition in that location attending only to interference condition $C_{j}$ is defined by:

$$
R A_{j}\left(u_{i}\right)= \begin{cases}1 & ; C_{j} \text { is true } \\ 0 & ; \text { otherwise }\end{cases}
$$

Hence, in a scenario with $U$ indoor locations, the percentage of reusable area attending to a given interference condition $C_{j}$ is denoted as $R A_{j}$ and computed as:

$$
R A_{j}(\%)=\frac{1}{U} \sum_{i=1}^{U} R A_{j}\left(u_{i}\right) \quad i=1, \ldots, U
$$

Finally, the percentage of the reusable area accounting for all interference conditions is obtained by:

$$
R A(\%)=\frac{1}{U} \sum_{i=1}^{U}\left(\prod_{j=1}^{4} R A_{j}\left(u_{i}\right)\right) \quad i=1, \ldots, U
$$

\section{B. Propagation Models.}

Fig. 3 shows the different radio propagation cases arisen in the considered scenarios for the computation of either interference or received power. For this we need to measure propagation loss in scenarios outdoor, indoor and building penetration.
Next, propagation models considered for each case are discussed in detail.

\section{- Building Penetration Losses}

The propagation model used to calculate the path loss between a transmitter located in the external part of a building and a receiver inside a room in other building, or vice versa, is given by the following expression:

$$
L=L_{\text {outside }}+W_{e}+W_{g e}+\max \left(W_{i} p, \alpha d_{w}\right)-h G_{h}
$$

where $L_{\text {outside }}$ is the loss from an external transmitter to the external part of the wall of any building, $W_{e}$ is the loss in the externally illuminated wall $(7 \mathrm{~dB}$, considering a penetration angle $\left.\phi=90^{\circ}\right), W_{g e}$ is the additional loss in the external wall for $\phi=0^{\circ}$ with a value of $4 \mathrm{~dB}$. $W_{i}$ is the loss in the internal walls $(6.9 \mathrm{~dB})$ and $p$ is the number of internal walls $(p=0,1,2 \ldots), \alpha$ is the loss per distance between adjacent walls $(0.6 \mathrm{~dB} / \mathrm{m})$ and $d_{w}$ is the distance among walls, $h$ is the height above the outdoor reference path loos level, and finally $G_{h}$ is the height gain $(1.6 \mathrm{~dB} / \mathrm{m})$ [6]. This model is used to compute the propagation loss $L_{p}$ of the interferences defined in (3), (4), (5) and (6). It is worth noting that the application of this model is done considering that transmitters and receivers are in different buildings.

\section{- Outside Propagation Model}

Using the model described in [7], we calculate the propagation loss between a transmitter and a receiver with different antenna heights. It also includes the case that the transmitter antenna height is less than or equal to receiver antenna height.

$$
\begin{aligned}
L_{\text {outside }}= & {\left[51-8 \log \left(H_{T X} H_{R X}\right)\right] \log (d)+8.4 \log \left(H_{T X} H_{R X}\right) } \\
& +20 \log \left(f_{c} / 2.2\right)+14
\end{aligned}
$$

where $d$ is the distance between transmitter and receiver, $H_{T X}$ is the transmitter's antenna height, $H_{R X}$ is the antenna height in the receiver; $f_{c}$ is the frequency. This model is used to obtain $L_{(B S-C P E)}$ and $L_{(C P E-B S)}$ in (1) and (2), respectively..

\section{- Indoor Propagation Model}

To model the propagation losses between a transmitter and a receiver located inside the same building, we used the model described in [6], as expressed by:

$L=L_{0}+L_{C}+\sum_{i=1}^{I} k_{w i} L_{w i}+k_{f}^{\left[\frac{k_{f}+2}{k_{f}+1}-b\right]} L_{f}$

where $L_{O}$ is the free space loss, $L_{C}$ is the constant loss fixed at $0 \mathrm{~dB}, L_{w i}$ is the loss of wall type $i(6.9 \mathrm{~dB}), L_{f}$ is the loss between adjacent floors $(18.3 \mathrm{~dB}), k_{w i}$ is the number of penetrated wall of type $i, k_{f}$ is the number of penetration floors $(1 \ldots 4), b$ is the empirical parameter fixed at 0.46. This model is used to compute the propagation loss $L_{p}$ of the interferences defined in $(3),(4),(5)$ and (6). It is worth noting that the application of 
this model is done considering that transmitters and receivers are in the same building.

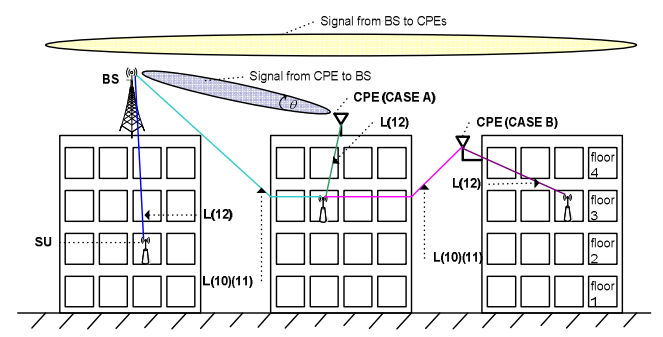

Fig. 3. Location of the systems, and use of the propagation models

\section{SimULATION RESULTS.}

In this section the reusable area is evaluated for Cases $\mathrm{A}$ and B identified in section II.B. In both cases, a Manhattan layout of $500 \times 500 \mathrm{~m}^{2}$ is considered with a total of 25 buildings uniformly distributed. Road's width is $20 \mathrm{~m}$. Buildings have 4 floors with a height of $2.5 \mathrm{~m}$ each. Each floor is $100 \times 100 \mathrm{~m}^{2}$ and has an internal distribution based on $5 \times 5 \mathrm{~m}$ compartments or rooms. Configuration parameters are shown in Table I. The protection margin $M$ is assumed to be the same for primary and secondary receivers and is fixed considering a shadowing margin given by $M_{S H}=16.4 \mathrm{~dB}$ and an interference margin given by $M_{I}=13.6 \mathrm{~dB}$. Notice that the considered shadowing margin allows to have an estimation of the interference levels $I_{i}, i=1 . .4$, stated in expressions (3), (4), (5) and (6) which is valid for the $95 \%$ of the cases under a lognormal shadowing characterization with a standard deviation of $10 \mathrm{~dB}$.

TABLE I

CONFIGURATION PARAMETERS

\begin{tabular}{|l|l|l|l|}
\hline \multicolumn{2}{|c}{ CONFIGURATION PARAMETERS } \\
\hline $\begin{array}{l}\text { Transmitter } \\
\text { Power, } P\end{array}$ & Obtained by (1) & Obtained by (2) & $15 \mathrm{dBm}$ \\
\hline Sensitivity, $S$ & $-80 \mathrm{dBm}$ & $-80 \mathrm{dBm}$ & $-65 \mathrm{dBm}$ \\
\hline $\begin{array}{l}\text { Protection } \\
\text { margin, } M\end{array}$ & $30 \mathrm{~dB}$ & $30 \mathrm{~dB}$ & $30 \mathrm{~dB}$ \\
\hline Antenna & Directional & Directional & $\begin{array}{l}\text { Omnidirec- } \\
\text { tional }\end{array}$ \\
\hline$F$ & $2 \mathrm{GHz}$ & $2 \mathrm{GHz}$ & $2 \mathrm{GHz}$ \\
\hline$G$ & $4 \mathrm{dBi}$ & $14 \mathrm{dBi}$ & $2 \mathrm{dBi}$ \\
\hline Fron/back ratio & $-30 \mathrm{~dB}$ & $-30 \mathrm{~dB}$ & $0 \mathrm{~dB}$ \\
\hline Antenna height & $12 \mathrm{~m}$ & $12 \mathrm{~m}$ & $1.5 \mathrm{~m} / \mathrm{floor}$ \\
\hline & & & \\
\hline \multicolumn{1}{|c|}{ CASE A } & & & $360^{\circ}$ \\
\hline HPBW Az & $120^{\circ}$ & $60^{\circ}$ & $360^{\circ}$ \\
\hline HPBW El & $20^{\circ}$ & $10^{\circ}$ & $360^{\circ}$ \\
\hline CASE B & & & $360^{\circ}$ \\
\hline HPBW Az & $180^{\circ}$ & $60^{\circ}$ & \\
\hline HPBW El & $20^{\circ}$ & $10^{\circ}$ & \\
\hline
\end{tabular}

\section{A. Case A.}

We initially analyze Case A assuming that the BS is on the flat roof of a building located in one of the corners of the Manhattan layout, while the CPEs are distributed on the flat roofs of the rest buildings. The transmission power of the BS is calculated using expression (1) and considering that coverage is provided to the roof of the farthest building with respect to the BS. This leads to a BS transmission power of $45.22 \mathrm{dBm}$.
Likewise, the transmission power of each CPE is calculated from (2) and depends on the location of each CPE within the considered scenario.

Fig. 4 shows the spatial availability of the primary band in each floor of the buildings. The white color in the figure represents the superposition of the zones where interference conditions $C_{1}$ to $C_{4}$ are fulfilled. On the other hand, the black color refers to the cases where interference conditions are not satisfied. Recall that these conditions are necessary to consider that there is spectrum reusability. As shown in Fig.4, spectrum availability is very limited in the $3^{\text {rd }}$ and $4^{\text {th }}$ floors due to the fact that: (a) higher floors are exposed to higher interference from the primary transmissions, and (b) the secondary transmission are prone to generate interference on primary receivers that are located in the roof's building. These reasons, along with the directivity of primary antennas cause interference concentrations in the highest floors. On the other hand, the reusable area is available inside of the buildings due to the following reasons: (a) transmissions of primary and secondary systems are highly attenuated by the walls and windows outside the buildings, so the interference inside of them decrease; (b) buildings located in the border of the layout do not receive interference from other CPEs in the outside part of the scenario; (c) in the buildings close to the BS because the CPEs on these buildings transmit with less power causing less interference.

Table II presents the percentage of reusable area for each interference condition as well as the superposition of all these conditions at each floor. We have observed that interference from the CPEs to SUs is the more restrictive interference condition, so the average over all floors of the corresponding reusable area (i.e. $R A_{2}$ ) is around $18 \%$. On the other hand, interference from SUs to the BS is the least restrictive interference which in turn leads to an average $R A_{3}$ of $73.69 \%$. In this context, the overall reusable area is mainly influenced by $R A_{2}$, so the average $R A$ is $17 \%$ less than $R A_{2}$. Note that the reusability difference between floor 1 and 4 is $39.31 \%$. As shown in Table 2, as we move vertically inside the buildings away from the primary transmitters the reusable area increases from $0 \%$ in the $4^{\text {th }}$ floor to $39.31 \%$ in the $1^{\text {st }}$ floor, given that the signals transmitted by the different systems have high propagation losses when these are crossing the floors.

Fig. 5 shows the how the $R A$ is affected by the protection margin considered for secondary users $\left(M_{S U}\right)$. We observe that for $M_{S U}$ values below of $15 \mathrm{~dB}$ the resulting $R A$ is constant because $I_{1}$ and $I_{2}$ decrease, while $I_{3}$ and $I_{4}$ do not change. The superposition of interferences is constant. In this situation primary receptors are more sensitive. For $M_{S U}$ values above of $20 \mathrm{~dB}$, the secondary receivers are more sensitive than primary receivers and the $R A$ decreases to approximately $0 \%$ at $50 \mathrm{~dB}$. Decreasing $M_{S U}$ from $50 \mathrm{~dB}$ to $25 \mathrm{~dB}$ the $R A$ increases $44.23 \%$ on floor 1 while floor 4 has $0 \%$ due to a high level of interference.

\section{B. Case B.}

In this case we have four BSs on flat roofs of the buildings located in the corners of the scenario layout, while CPEs are assumed to be on external windows of the other buildings. 
Similarly than the previous case, the transmission power of BSs is calculated by means of (1) resulting in a power value of $43.81 \mathrm{dBm}$. We consider that the signal from each BS in the corner communicate with a CPE located in lower external windows of the central building. The transmission power of the $\mathrm{CPE}$ is calculated using expression (2), considering that each $\mathrm{CPE}$ is directed to the $\mathrm{BS}$ with better reception. We uniformly distribute $96 \mathrm{CPEs}$ in the scenario, resulting in an average of 24 CPEs per coverage zone of each BS using 20 snapshots for each CPE set. The percentages of reusable area for Case B are shown in Table III. Similar than in Case A, the average reusable area over all floors is more influenced by $R A_{2}$ than RA3, with an average of $24.30 \%$ and $49.88 \%$, respectively. The average of $R A$ is $15.95 \%$, which is similar to the one obtained in Case A. The difference is that in case B the average value of $R A$ is computed over all floors.

\section{CONCLUSIONS.}

In this paper we have quantified the percentage of locations where it is possible to exploit OSA for secondary short-range radio communication systems within indoor locations in dense urban areas. The spectrum band to be reused is that of a primary system that provides outdoor coverage within the considered area. The analysis have been conducted assuming a typical building layout for dense urban areas and relying on well-know propagation models to characterize outdoor, outdoor to indoor and indoor propagation. In particular, four different conditions for spectrum reusability have been identified according to the different types of interferences arisen in the scenario. Results demonstrate the amount and spatial distribution of the reusable area attending to different system configuration parameters. As future work, we envisage to compare obtained results with real measurements.

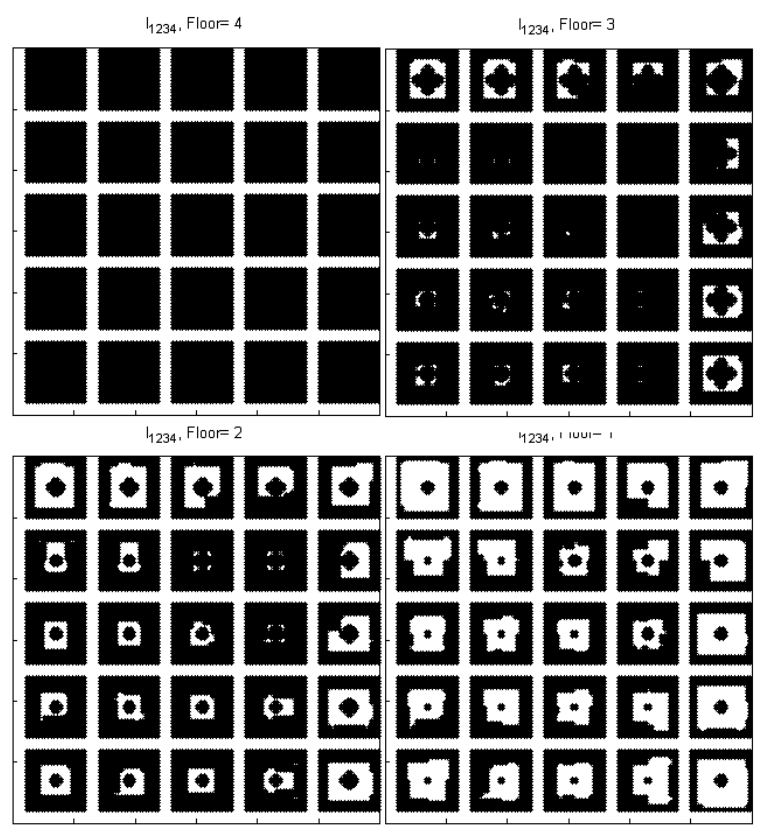

Fig. 4. Spatial availability of the primary band inside the buildings. Case A.

TABLE II

PERCENTAGE OF REUSABLE AREA FOR CASE A

\begin{tabular}{|c|c|c|c|c|}
\hline & Floor $_{1}$ & Floor $_{2}$ & Floor $_{3}$ & Floor $_{4}$ \\
\hline$R A_{1}$ & 67.82 & 47.64 & 35.56 & 24.96 \\
\hline$R A_{2}$ & 41.44 & 22.06 & 9.51 & 0.63 \\
\hline$R A_{3}$ & 94.35 & 82.45 & 66.04 & 51.93 \\
\hline$R A_{4}$ & 61.33 & 39.44 & 22.13 & 2.71 \\
\hline$R A$ & 39.31 & 20.01 & 7.46 & 0 \\
\hline
\end{tabular}

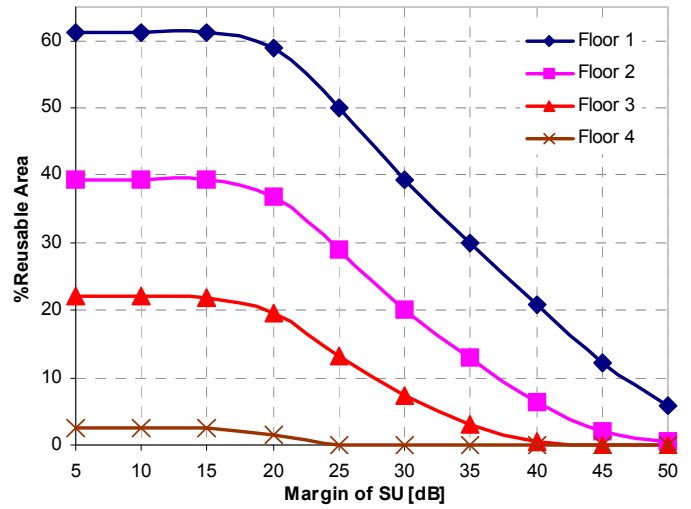

Fig. 5. Reusable area percentage versus the protection margin of secondary users $\left(M_{S U}\right)$ in Case A.

TABLE III

PERCENTAGE OF REUSABLE AREA FOR CASE B

\begin{tabular}{|l|l|l|l|l|}
\hline & Floor $_{1}$ & Floor $_{2}$ & Floor $_{3}$ & Floor $_{4}$ \\
\hline$R A_{1}$ & 45.04 & 29.4 & 19.6 & 9.92 \\
\hline$R A_{2}$ & 37.63 & 26.15 & 18.94 & 14.5 \\
\hline$R A_{3}$ & 73.48 & 56.08 & 42.24 & 27.72 \\
\hline$R A_{4}$ & 41.99 & 27.76 & 19.73 & 15.08 \\
\hline$R A$ & 28.89 & 17.86 & 10.38 & 6.69 \\
\hline
\end{tabular}

\section{ACKNOWLEDGEMENT.}

The authors wish to acknowledge the activity of the Network of Excellence in Wireless COMmunications NEWCOM++ of the European Commission (contract n. 216715) that motivated this work. This work was supported in part by CYCIT (Spanish National Science Council) under grant TEC2006-09109 at UPC.

\section{REFERENCES.}

[1] Qing Zhao; Sadler, B.M., "A Survey of Dynamic Spectrum Access," Signal Processing Magazine, IEEE, vol.24, no.3, pp.79-89, May 2007.

[2] Senhua Huang; Xin Liu; Zhi Ding, "Opportunistic Spectrum Access in Cognitive Radio Networks," INFOCOM 2008. The 27th Conference on Computer Communications. IEEE , vol., no., pp.1427-1435, 13-18 April 2008.

[3] K. Nishimori, E. Bottega, H. Yomo, P. Popovski, Y. Takatori, R. Prasad, and S. Kubota, "Spatial availability for cognitive radio system under directional interference," in Proc. 9th International Symposium on Wireless Personal Multimedia Communications (WPMC'06), Sep. 2006.

[4] Nishimori, K.; Di Taranto, R.; Yomo, H.; Popovski, P.; Takatori, Y.; Prasad, R.; Kubota, S., "Spatial Opportunity for Cognitive Radio Systems with Heterogeneous Path Loss Conditions," Vehicular Technology Conference, 2007. VTC2007-Spring. IEEE 65th, vol., no., pp.2631-2635, 22-25 April 2007

[5] X.Jing, S.Mau, D. Raychaudhuri, R.Matyas, "Reactive cognitive radio algorithms for Co-existence between IEEE $802.11 \mathrm{~b}$ and $802.16 \mathrm{a}$ networks," IEEE Global Telecommunications Conference (GLOBECOM '05), vol.5, pp.2465 - 2469, Nov./Dec., 2005.

[6] COST (European Co-operation in the Field of Scientific and Technical Research), COST 231 Book, Final Report. Chapter 4, Propagation Prediction Models.

[7] S. Ichitsubo, et al.,"2GHz-Band Propagation Loss Prediction in Urban areas; antenna heights ranging from ground to building roof," IEICE Technical Report, A·P 96-15, May, 1996. 